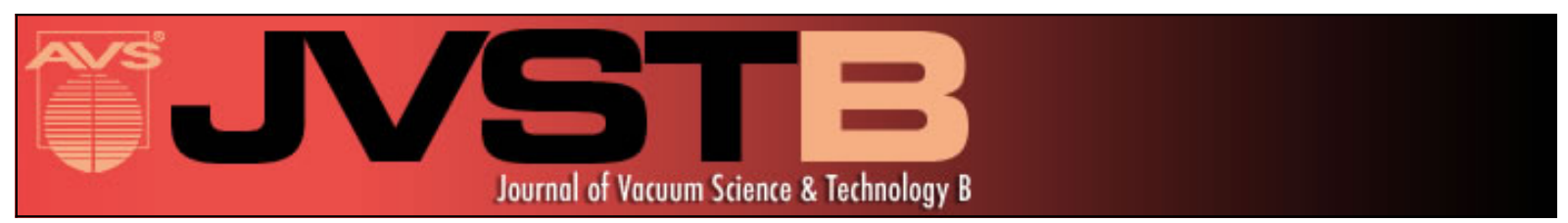

\title{
Field emission beam characteristics of single metal nanotip cathodes with on-chip collimation gate electrode
}

Chiwon Lee, Pratyush Das Kanungo, Vitaliy Guzenko, Patrick Hefenstein, R. J. Dwayne Miller, and Soichiro Tsujino

Citation: Journal of Vacuum Science \& Technology B 33, 03C111 (2015); doi: 10.1116/1.4913397

View online: http://dx.doi.org/10.1116/1.4913397

View Table of Contents: http://scitation.aip.org/content/avs/journal/jvstb/33/3?ver=pdfcov

Published by the AVS: Science \& Technology of Materials, Interfaces, and Processing

\section{Articles you may be interested in}

Ion-beam morphological conditioning of carbon field emission cathode surfaces

J. Vac. Sci. Technol. B 23, 731 (2005); 10.1116/1.1880052

Emission characteristics of $\mathrm{NbC/Nb}$ field emitter array cathodes

J. Vac. Sci. Technol. B 21, 422 (2003); 10.1116/1.1527636

Field emission cathode array with self-aligned gate electrode fabricated by silicon micromachining

J. Vac. Sci. Technol. B 18, 3544 (2000); 10.1116/1.1324648

Effects of thermal annealing on emission characteristics and emitter surface properties of a Spindt-type field emission cathode

J. Vac. Sci. Technol. B 16, 783 (1998); 10.1116/1.590218

Novel structure of a silicon field emission cathode with a sputtered TiW gate electrode

J. Vac. Sci. Technol. B 16, 242 (1998); 10.1116/1.589788

\section{HIDEN}

\section{Instruments for Advanced Science}

Contact Hiden Analytical for further details: w www.HidenAnalytical.com E info@hiden.co.uk CLICK TO VIEW our product catalogue

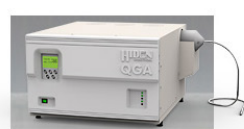

Gas Analysis

dynamic measurement of reaction gas streams catalysis and thermal analysis

molecular beam studies

'

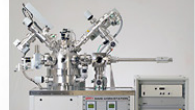

Surface Science

UHVTPD

SIMS elemental imaging - surface mapping

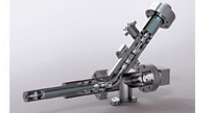

Plasma Diagnostics plasma source characterization etch and deposition process reaction kinetic studies analysis of neutral and radical species

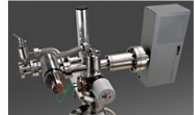

Vacuum Analysis partial pressure measurement and control of process gases reactive sputter process contro , vacuum diagnostics vacuum coating process monitoring 


\title{
Field emission beam characteristics of single metal nanotip cathodes with on-chip collimation gate electrode
}

\author{
Chiwon Lee L) $^{\text {a) }}$ \\ Laboratory for Micro and Nanotechnology, Paul Scherrer Institute, Villigen 5232, Switzerland \\ and Max Planck Institute for Structure and Dynamics of Matter, CFEL (Bld. 99), \\ Luruper Chaussee 149, 22761 Hamburg, Germany \\ Pratyush Das Kanungo, Vitaliy Guzenko, and Patrick Hefenstein \\ Laboratory for Micro and Nanotechnology, Paul Scherrer Institute, Villigen 5232, Switzerland \\ R. J. Dwayne Miller \\ Max Planck Institute for Structure and Dynamics of Matter, CFEL (Bld. 99), Luruper Chaussee 149, \\ 22761 Hamburg, Germany and Departments of Chemistry and Physics, University of Toronto, \\ 80 St. George Street Toronto, Ontario M5S 3H6, Canada \\ Soichiro Tsujino ${ }^{\text {b) }}$ \\ Laboratory for Micro and Nanotechnology, Paul Scherrer Institute, Villigen 5232, Switzerland
}

(Received 8 December 2014; accepted 4 February 2015; published 26 February 2015)

\begin{abstract}
Field-emission and beam collimation characteristics of single metal nanotip devices with doublegate electrodes are studied. Applying a previously developed method to fabricate all-metal doublegate nanotip arrays with a stacked on-chip extraction $G_{\mathrm{ext}}$ and collimation $G_{\mathrm{col}}$ gate electrodes with the large $G_{\mathrm{col}}$ apertures, the authors produced single double-gate nanotip devices and measured their beam characteristics. Excellent beam collimation capability with minimal reduction of the emission current and the enhancements of the current density up to a factor of $\sim 7$ was observed. The results indicate that these single nanotip devices are highly promising for electron beam applications that require extremely high brilliance and coherence. (C) 2015 American Vacuum Society.

[http://dx.doi.org/10.1116/1.4913397]
\end{abstract}

\section{INTRODUCTION}

Ultrabright cathodes are critical elements in a broad range of applications such as cathodes for $\mathrm{x}$-ray free electron lasers as well as time resolved electron microscopy and diffraction experiments that aim at resolving the dynamics of materials and molecules on the atomic length and time scales. One of the core requirements for such cathodes is the generation of a low emittance electron beam with high current to ensure the delivery of sufficient current to the target. For such applications, metal nanotip field emitters ${ }^{1-8}$ are attracting considerable interest recently as high brilliance cathodes that may outperform state-of-the-art photocathodes. This is because metal nanotips allow high emission current with narrow energy spread via quantum tunnelling of electrons through the surface barrier at the nanometer scale emitter tip apexes. One can also generate ultrafast electron pulses by exciting the metal nanotips with ultrafast laser pulses. ${ }^{1-7}$ So far, etched-wire needle-shaped field emitters prepared by electrochemical etching and in-situ thermal treatment have been the most widely studied. ${ }^{1-5}$ However, for high charge, high beam brilliance applications, the cathode must be used under a high acceleration electric field, on the order of $10-100 \mathrm{MV} / \mathrm{m}$, i.e., the maximum extraction field conditions for minimum spacecharge broadening to conserve the beam brilliance with the highest bunch density possible. This makes the nanofabricated metal nanotip emitters with on-chip gate electrodes

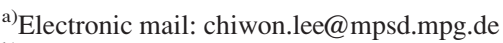

${ }^{\mathrm{b})}$ Electronic mail: soichiro.tsujino@psi.ch
}

attractive for many applications since the electron emission can be switched with the application of a low gate potential on the order of $100 \mathrm{~V}$ independently from the acceleration field. ${ }^{9,10}$ In fact, integration of the single-gate field emission array (FEA) into a combined diode-RF cavity electron gun and stable subnanosecond pulsed field emission operation under the acceleration field up to $30 \mathrm{MV} / \mathrm{m}$ has been reported recently. ${ }^{9}$

Further, highly collimated electron beams can be generated from double-gate FEAs by adding a collimation gate electrode on top of the electron extraction gate electrode and individually collimating the field emission beamlet. ${ }^{11} \mathrm{We}$ note that this is crucial to generate the high intrinsic brilliance FEA beam. ${ }^{12,13}$ The intrinsic transverse beam emittance of a single beamlet, given by the root-mean-square (rms) radius $R_{\text {tip }}$ of the emitter tip apex and the angular divergence equal to $\sim 30^{\circ}$, is on the order of $10^{-3} \mathrm{~mm} \mathrm{mrad}$. This extremely small value is a consequence of the small $R_{\text {tip }}$ in the nanometer range. In the case of single-gate FEAs with the large target current of several amperes, the transverse beam emittance is on the order of $1 \mathrm{~mm}$ mrad. However, the transverse emittance can be much reduced with double-gate FEAs. By decreasing the angular beam divergence of the individual beamlet by an order of magnitude, the double-gate FEA beam can potentially achieve an intrinsic emittance lower than that of state-of-the-art high brilliance photocathode (equal to $\sim 0.2 \mathrm{~mm} \mathrm{mrad}$ with the cathode radius of $0.5 \mathrm{~mm}$ ).

The excellent beam collimation capability of double-gate FEAs with a large collimation gate aperture was 


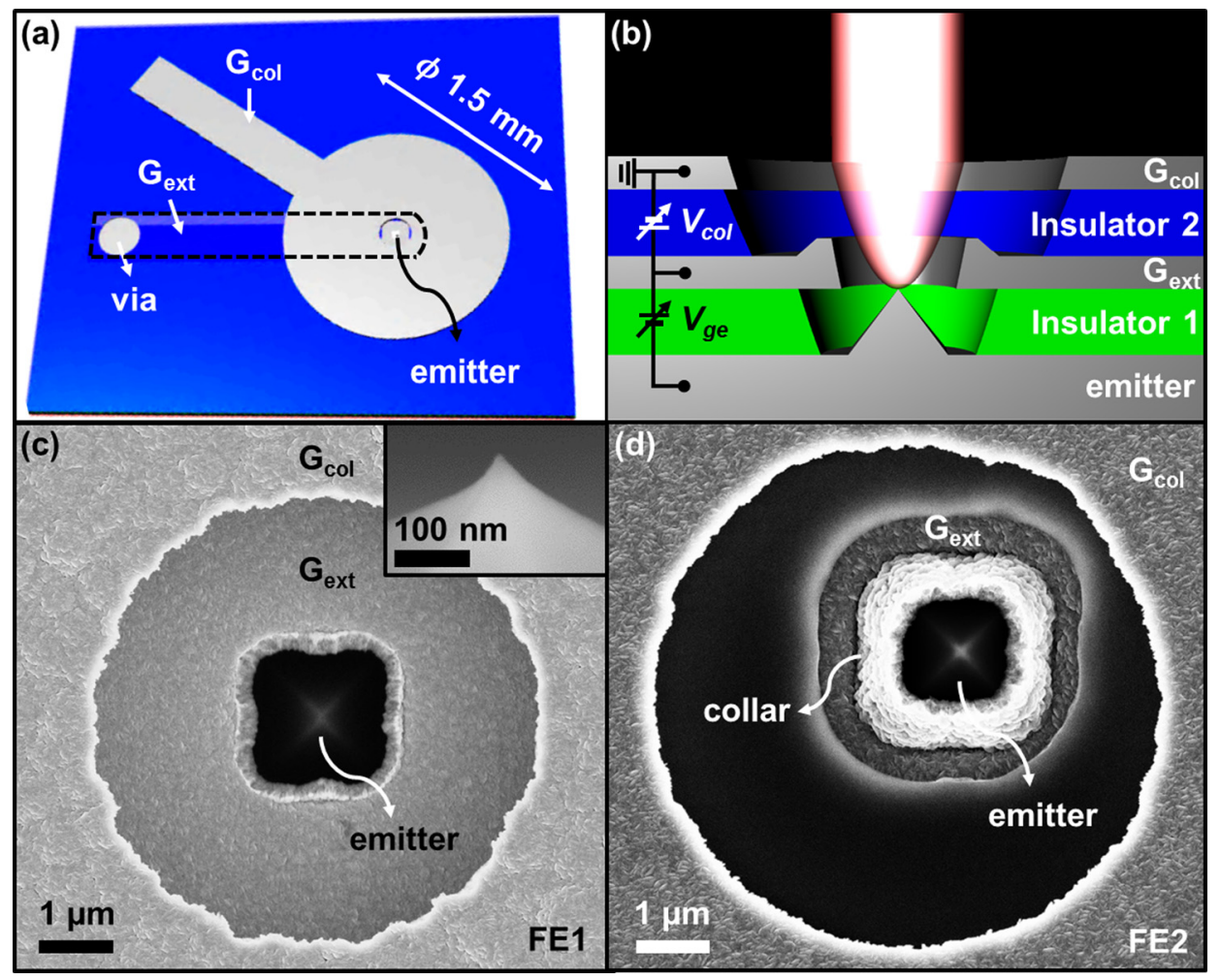

FIG. 1. (Color online) (a) Top-view and (b) cross-sectional-view schematic diagram of the double-gate single nanotip emitter device. In (a), the dotted line indicates the $G_{\text {ext }}$ electrode buried underneath the insulator 2 . The electrical contact to $G_{\text {ext }}$ is provided through via hole through the insulator 2 . The $G_{\mathrm{col}}$ electrode is a $1.5 \mathrm{~mm}$-diameter circular shape with a rectangular contact pad. In (b), the schematic trajectory (envelope) of the maximally collimated beam with $V_{\text {col }}$ equal to $\sim\left(-V_{\text {ge }}\right)$ is shown. (c) and (d) show the SEM image of FE1 and FE2, respectively. Inset of (c) shows the high magnification SEM image of emitter tip apex of FE1 observed with the viewing angle of $47^{\circ}$.

experimentally demonstrated recently. ${ }^{13}$ An order of magnitude enhanced beam intensity was observed from a $4 \times 10^{4}$ tip double-gate FEA at the maximally collimated condition. Together with the compatibility with high acceleration electric field extraction and the possibility to generate ultrafast electron pulses using near infrared ultrafast laser excitation, these FEAs are highly promising as ultrabright electron sources that may open up new applications in femtosecond time resolved applications.

In this work, we study the field emission and beam collimation properties of single nanotip double-gate emitters to investigate the characteristics of the individual nanotips that were only inferred in the previous experiments on arrays. Such investigation is important on one hand to further improve the beam collimation properties and beam uniformity for scaling to arrays, and on the other hand, to explore the applicability of such metal nanotips for high brilliance beam applications.

\section{SAMPLE AND EXPERIMENT}

Two double-gate single nanotip devices (FE1 and FE2), see Fig. 1 and Table I, studied in this work were fabricated by adapting the fabrication method for arrays of double-gate emitters. ${ }^{14,15}$ It starts with the preparation of molybdenum nanotips by the molding method using the oxidized $\mathrm{Si}$ wafer where pyramidal pits are patterned on its surface. The emitters are pyramidal shape with $\sim 1.5 \mu \mathrm{m}$ base size and with the emitter tip apex radius of curvature in the range of 5-10 nm, see Fig. 1(c) inset. On top of the emitter substrate, the extraction gate electrode $G_{\text {ext }}$ and the collimation gate electrode $G_{\mathrm{col}}$ were fabricated. The gate electrodes were patterned from $0.5 \mu \mathrm{m}$-thick molybdenum layers. The emitter substrate and $G_{\text {ext }}$ are separated by a $\sim 1.2 \mu \mathrm{m}$-thick $\mathrm{SiO}_{2}$ layer, and $G_{\text {ext }}$ and $G_{\text {col }}$ are separated by a $\sim 1.2 \mu \mathrm{m}-$ thick $\mathrm{SiON}$ layer. The $G_{\text {ext }}$ apertures were patterned by the self-aligned process. A collar structure surrounds the $G_{\text {ext }}$ aperture edge, which is height-controllable by adjusting mask-etching and wet-etching duration of the self-aligned $G_{\text {ext }}$ aperture patterning step. The $G_{\mathrm{col}}$ apertures with 3-5 times larger aperture diameter than that of $G_{\text {ext }}$ were patterned by electron beam lithography. On a $7 \mathrm{~mm}$-square chip, six such double-gate single nanotips were fabricated. For the experiment, each chip was mounted on a sample holder, wire-bonded for the gate contacts with Al-wires with

TABLE I. Structural parameters of FE1 and FE2. The lateral offset of the emitter is measured from the center of $G_{\mathrm{col}}$ aperture.

\begin{tabular}{lcc}
\hline \hline & FE1 & FE2 \\
\hline$G_{\text {ext }}$ aperture diameter $(\mu \mathrm{m})$ & 1.69 & 1.43 \\
$G_{\text {ext }}$ collar thickness $(\mu \mathrm{m})$ & 0.25 & 0.56 \\
$G_{\text {col }}$ aperture diameter $(\mu \mathrm{m})$ & 5.97 & 6.85 \\
Aperture diameter ratio & 3.5 & 4.8 \\
Emitter lateral offset $(\mu \mathrm{m})$ & 0 & 0.8 \\
\hline \hline
\end{tabular}


the diameter of $20 \mu \mathrm{m}$, and loaded into a measurement chamber equipped with a phosphor screen and a retractable Faraday cup (FC) with a background pressure of $(0.5-1.5) \times 10^{-8}$ mbar.

The field emission beam was measured as a function of the electron extraction potential $V_{\mathrm{ge}}$ and the beam collimation potential $V_{\text {col }}$ under DC or pulsed conditions. When a negative collimation potential $V_{\mathrm{col}}$ is applied between $G_{\mathrm{col}}$ and $G_{\text {ext }}$ while generating the field emission beam by applying a positive electron extraction potential $V_{\text {ge }}$ between $G_{\text {ext }}$ and emitter, see Fig. 1(b), the angular divergence of the beam is substantially reduced. In the case of the DC measurements, we connected $G_{\text {ext }}$ to ground potential and applied $-V_{\text {ge }}$ to the emitter substrate and $V_{\text {col }}(<0)$ to $G_{\text {col }}$. The DC gate potentials were applied using source-measure units (Keithley 2400) and scanning the voltages with $20 \mathrm{~ms}$ steps. For the pulse measurement, we connected $G_{\mathrm{col}}$ to ground and applied $-V_{\text {col }}$ to $G_{\text {ext }}$ and $\left(-V_{\text {ge }}+\left|V_{\text {col }}\right|\right)$ to the emitter substrate. The pulsed potential, with a typical duration of $200 \mu$ s, was generated using a custom-built double-pulser. The field emission current was measured either directly by inserting the FC in front of the device with a separation of $10 \mathrm{~mm}$ or from the integrated beam intensity of the beam images observed on the phosphor screen (calibrated by the current measured with the FC). The beam image was measured with the phosphor screen at the screen potential of $2.5 \mathrm{kV}$ when the device was placed $50 \mathrm{~mm}$ from the screen. For the beam measurements, we captured the phosphor screen image generated by pulsed field emission using a synchronously triggered CCD camera with an effective resolution of 16-bit. Care was taken to avoid saturation of all pixel values to be able to evaluate the relative field emission current of the beam as well as the rms radius denoted by dashed white circle in the images in Figs. 3 and 4. The emission current and beam image data presented below were recorded after several hours of conditioning that was done by scanning $V_{\text {ge }}$ between 0 and $100 \mathrm{~V}$ until the field emission current-voltage characteristics became stable.

\section{RESULTS AND DISCUSSION}

Figure 2(a) shows the relation between the field emission current $I$ and $V_{\text {ge }}$ at zero $V_{\text {col }}$ for FE1 and FE2. The observed $I-V_{\mathrm{ge}}$ characteristics of the devices above the noise level $(\sim 0.5 \mathrm{nA}$ for the FE1 measurement and $\sim 0.2 \mathrm{nA}$ for the FE2 measurement) were described well by the equation

$$
I=A_{\mathrm{FN}}\left(V_{\mathrm{ge}} / B_{\mathrm{FN}}\right)^{2} \exp \left(-B_{\mathrm{FN}} / V_{\mathrm{ge}}\right),
$$

as shown by the curves in Fig. 2(a). The fitting parameters $A_{\mathrm{FN}}$ and $B_{\mathrm{FN}}$ were equal to $1.6 \times 10^{-4} \mathrm{~A}$ and $1000 \mathrm{~V}$ for FE1, and 2.95 A and 997 V for FE2, respectively. The same $B_{\mathrm{FN}}$ values of the two emitters (within $\sim 10 \%$ of the estimated uncertainty) indicate the stability of the tip fabrication process since $B_{\mathrm{FN}}$ is largely determined by the tip apex radius of curvature $R_{\text {tip }}$; from the comparison of the observed $B_{\mathrm{FN}}$ value and the calculated emission characteristic as a function of $R_{\mathrm{tip}}{ }^{16}$ we found that the estimated $R_{\mathrm{tip}}$ are equal
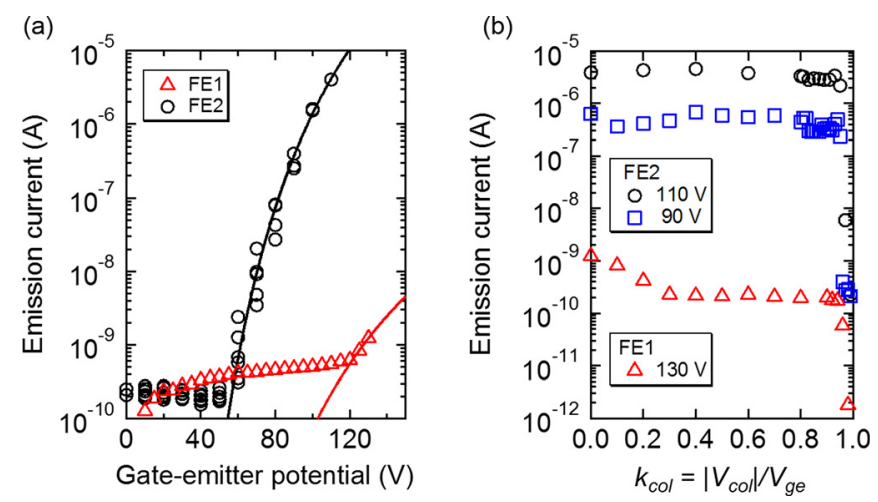

FIG. 2. (Color online) Field emission characteristics of double-gate single nanotip emitters. (a) The relations between the field emission current and $V_{\text {ge }}$ at zero collimation potential for FE1 (triangles) and FE2 (circles). Curves are the fitted result of the data above the noise level with the Fowler-Nordheim function. (b) The relations between the field emission current and the collimation potential ratio $k_{\mathrm{col}}$.

to $\sim 10 \mathrm{~nm}$ for both devices that is consistent with the highresolution SEM image of the emitter tip apex [Fig. 1(c), inset].

In Fig. 2(b), we show the field emission current at finite $V_{\text {col }} . V_{\text {ge }}$ was fixed at $130 \mathrm{~V}$ for FE1 (open triangles). For FE2, two sets of measurements with $V_{\text {ge }}$ of $90 \mathrm{~V}$ (open squares) and $110 \mathrm{~V}$ (open circles) are displayed. In Fig. 2(b), we denote $V_{\text {col }}$ by the ratio $k_{\text {col }}$ defined as

$$
k_{\mathrm{col}} \equiv-V_{\mathrm{col}} / V_{\mathrm{ge}} \text {. }
$$

In the case of FE1, the emission beam current was diminished to $18 \%$ of the uncollimated beam current $\left(k_{\mathrm{col}}=0\right)$ already when $k_{\mathrm{col}}$ was equal to 0.3 , implying that much of the electric field at the emitter tip apex applied via $V_{\text {ge }}$ is relaxed by $V_{\text {col }}$. Increasing $k_{\mathrm{col}}$ to 0.94 resulted in the emission current equal to $4.7 \%$ of the uncollimated beam current. Further increase of $k_{\text {col }}$ to 0.96 results in the decrease of the emission current by several orders of magnitude. In contrast, in the case of FE2, more than $70 \%$ of the uncollimated beam current was maintained at $k_{\mathrm{col}}$ of 0.94 . At the same value, the peak current density was maximum as shown in Fig. 5. We ascribe the improved current conservation characteristic of FE2 to the structural features of gate apertures of the device; compared to those of FE1, the $\sim 400 \mathrm{~nm}$-taller-collar in height, surrounding $G_{\text {ext }}$ aperture, together with the $\sim 1 \mu \mathrm{m}$-larger- $G_{\mathrm{col}}$ aperture diameter provide additional electrostatic shielding of the emitter tip apex by preventing direct line-of-sight from the tip apex from $G_{\mathrm{col}}$ aperture, and reducing the influence of $V_{\mathrm{col}}$ on the emission current.

Figure 3 presents the selected field emission beam images of FE1 for $k_{\text {col }}$ between 0 and 0.98 when $V_{\text {ge }}$ was equal to $143 \mathrm{~V}$. The uncollimated beam with zero $k_{\mathrm{col}}$ showed two separated spots with different beam intensities. Interestingly, as we increased $k_{\mathrm{col}}$, the two spots converged as indicated by the shrinking beam envelopes denoted by the broken circles toward point $\mathrm{C}$, given by the crossing of the horizontal and vertical broken lines. This suggests that only a part of the emitter apex within the radius of $R_{\text {tip }}$ is active, but $G_{\mathrm{col}}$ can collimate the beam emitted from the whole apex. When $k_{\text {col }}$ 

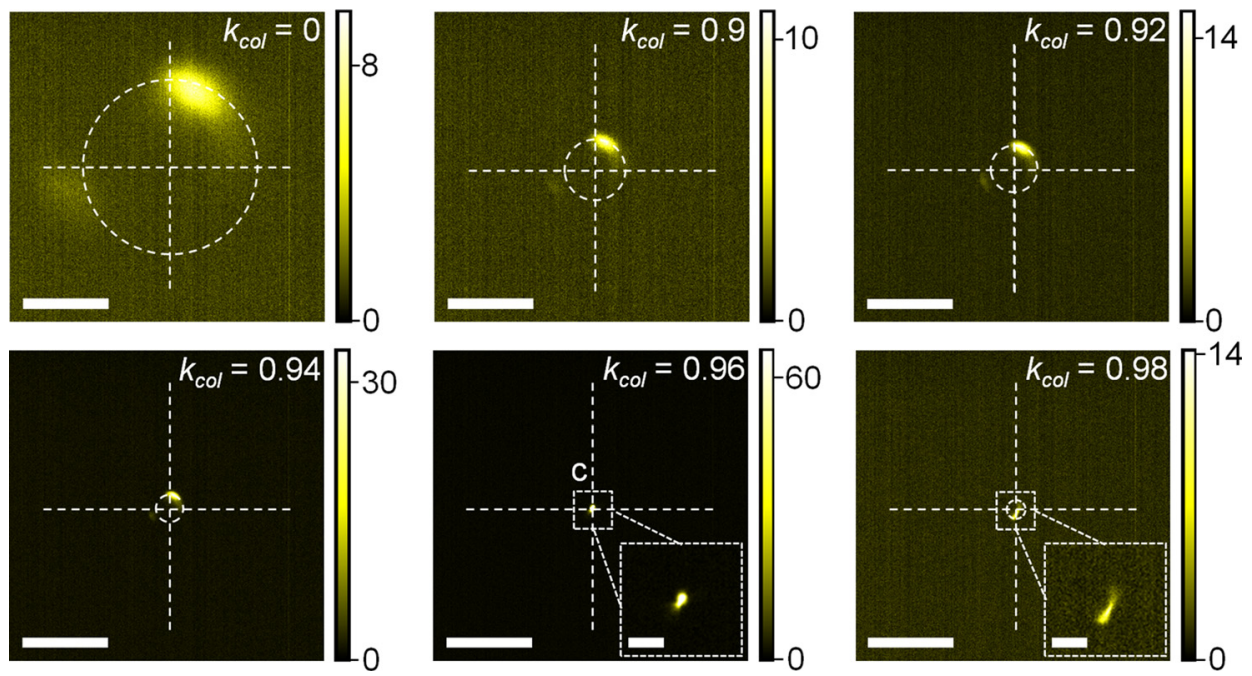

FIG. 3. (Color online) Variation of the field emission beam of FE1 with the increase of $k_{\mathrm{col}}$ from 0 to 0.98 for $V_{\mathrm{ge}}$ of $143 \mathrm{~V}$. The scale bar of the images is $5 \mathrm{~mm}$. The inset images are enlarged to highlight the small beam spot (the scale bar is $1 \mathrm{~mm}$.).

was equal to 0.96 , the two spots became indistinguishable at point $\mathrm{C}$ and the peak beam intensity was enhanced by a factor of $\sim 7$ from the uncollimated beam with $k_{\mathrm{col}}$ of 0 . Further increase of $k_{\mathrm{col}}$ to 0.98 substantially reduced the emission current and beam intensity. The comparison of the two beams with $k_{\mathrm{col}}$ equal to 0.96 and 0.98 suggests the overfocusing of the beam at $k_{\mathrm{col}}$ of 0.98 .

Figure 4 shows the field emission beam of FE2 at various $k_{\mathrm{col}}$ for $V_{\mathrm{ge}}$ equal to $90 \mathrm{~V}$. Differently from FE1, the zero $k_{\mathrm{col}}$ field emission beam of FE2 spreads more uniformly over the area with the diameter of $\sim 5 \mathrm{~mm}$, thereby indicating that much larger fraction of the emitter tip apex within $\mathrm{R}_{\text {tip }}$ contributes to the field emission. When we increased $k_{\mathrm{col}}$ from 0 to 0.8 , the beam size reduced only gradually by $\sim 25 \%$. Then further increase of $k_{\mathrm{col}}$ to 0.94 resulted in the steep reduction of the beam by a factor of 10 with the simultaneous increase of the peak beam intensity by a factor of $\sim 6$, see Fig. 5 . A similar beam collimation was also observed with the high current beam at $V_{\mathrm{ge}}$ of $110 \mathrm{~V}$. These observations demonstrate the excellent beam collimation characteristic of these double-gate single nanotip emitters. ${ }^{17}$

In Fig. 6, we summarized the relation between the beam radius and $k_{\mathrm{col}}$. From the rms beam radius $R$, we evaluated the rms transverse velocity $u_{t}$ with the assumption of free propagation of the electrons in the transverse direction while they are accelerated along the beam axis, see Ref. 8. In short, $u_{t}$ is equal to $\sim R / T$, where $T$ is the propagation time of the electron from the emitter to the screen. $T$ is approximately equal to $2 L / u_{\mathrm{scr}}$, where $L$ is the emitter-screen distance equal to $50 \mathrm{~mm}$ and $u_{\mathrm{scr}}$ is the velocity equal to $\sqrt{2 e V_{\mathrm{scr}} / m_{e}}\left(V_{\mathrm{scr}}\right.$ is the screen potential equal to $2.5 \mathrm{kV}, e$ is the electron charge, and $m_{e}$ is the electron rest mass). Figure 6 shows an order of magnitude reduction of $u_{t}$ with the increase of $k_{\mathrm{col}}$ from zero to 0.94 (FE1 case) or 0.95 (FE2 case) with the smallest value compatible with the array beam experiment. ${ }^{8,18}$
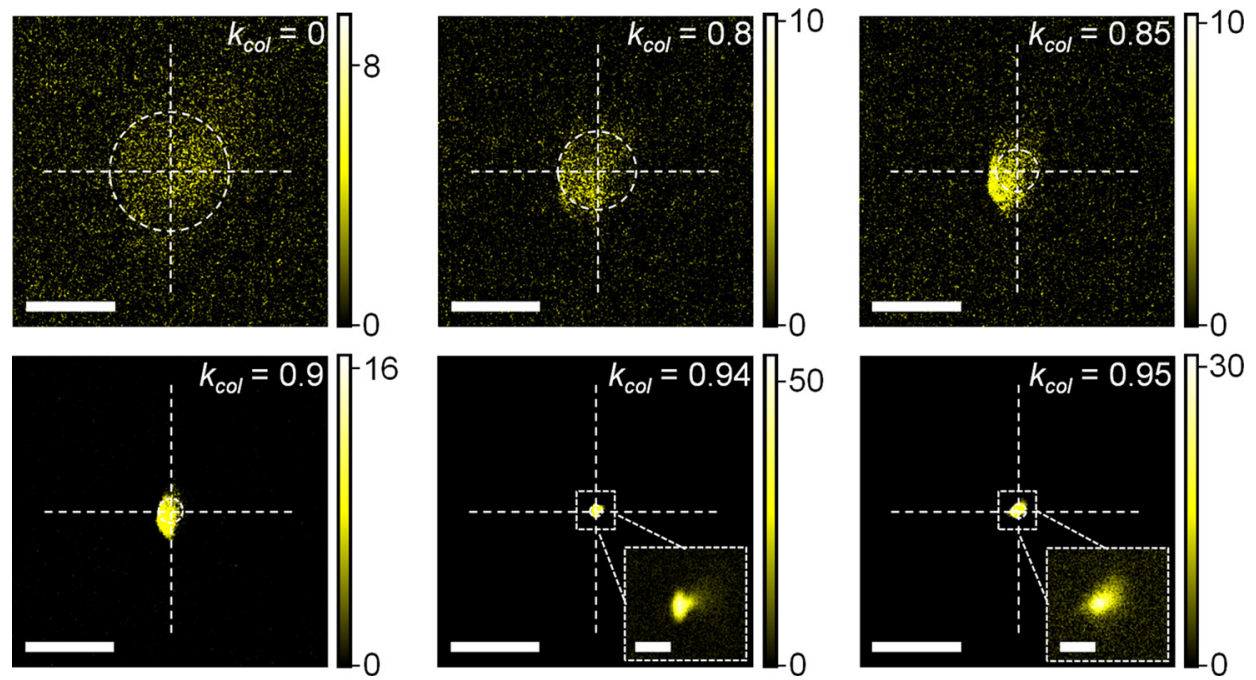

FIG. 4. (Color online) Variation of the field emission beam of FE2 with the increase of $k_{\text {col }}$ from 0 to 0.95 for $V_{\text {ge }}$ of $90 \mathrm{~V}$. The scale bar of the images is 5 mm. The inset images are enlarged to highlight the small beam spot (the scale bar is $1 \mathrm{~mm}$.). 


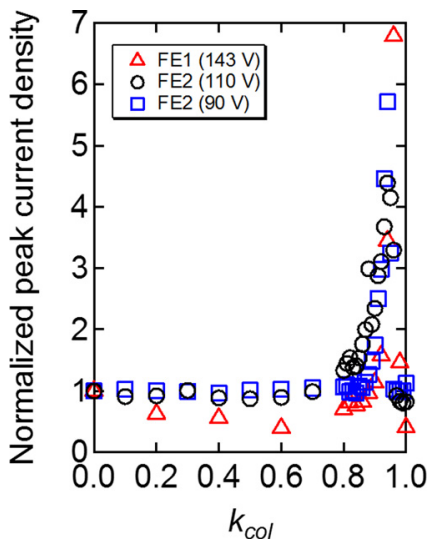

FIG. 5. (Color online) Peak current density of the field emission beams as a function of $k_{\mathrm{col}}$. The current density values were normalized by the zero $k_{\mathrm{col}}$ value of each scan.

As the beam images in Fig. 6 show, we found that $R$ and $u_{t}$ of FE2 beam at $k_{\text {col }}$ of 0.94 increased by a factor 3 when $V_{\text {ge }}$ was increased from 90 to $110 \mathrm{~V}$ with the concomitant increase of the emission current from $\sim 50 \mathrm{nA}$ to $\sim 0.5 \mu \mathrm{A}$. This effect is likely caused by the Coulomb repulsion of the electrons. In the case of the uncollimated beam, the large beam divergence at the source (on the order of $30^{\circ}$ ) quickly dilutes the density of electrons as they propagate to the anode (screen), and the beam radius on the screen is unchanged within $20 \%$ as the current increases from $\sim 100 \mathrm{nA}$ to $\sim 4 \mu \mathrm{A}$. However, such a dilution does not occur in the case of the highly collimated beam with $k_{\mathrm{col}}$ of $\sim 1$ because of the order of magnitude smaller initial angular spread. Therefore, the initial diameter of the beam (equal to at most the diameter of the $G_{\text {col }}$

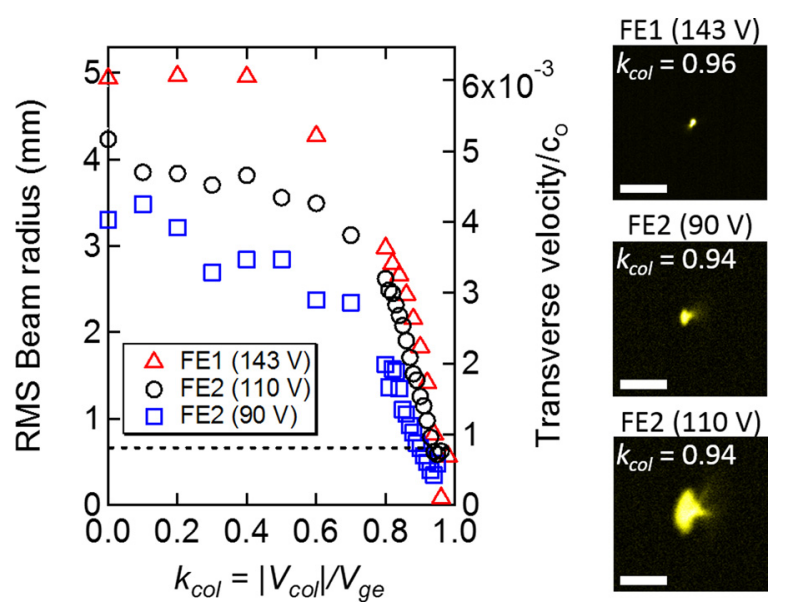

FIG. 6. (Color online) Variation of the rms radius of the beam envelope and transverse velocity $u_{t}$ normalized by the light velocity in vacuum $c_{0}$ with the increase of $k_{\text {col }}$ from 0 to $\sim 1$. For each scan, $V_{\text {col }}$ was varied while $V_{\text {ge }}$ was fixed at certain value; for FE1 $V_{\text {ge }}$ was equal to $143 \mathrm{~V}$ (red triangle), and for $\mathrm{FE} 2, V_{\text {ge }}$ was equal to $90 \mathrm{~V}$ (blue squares) and $110 \mathrm{~V}$ (black circles). The broken line shows the transverse velocity equal to $8 \times 10^{-4} c_{0}$, corresponding to the performance of the state-of-the-art photocathode with intrinsic beam emittance of $0.2 \mathrm{~mm}$ mrad for a $1 \mathrm{~mm}$-diameter cathode currently in use. The maximally collimated beams of each scans are also shown. The scale bar of the images corresponds to $2 \mathrm{~mm}$. aperture) at a few micron above the $G_{\text {col }}$ plane can be maintained on the screen only when the beam current density is small or when the acceleration field is sufficiently large so that the longitudinal velocity is increased quickly before the space-charge expansion of the beam increases $u_{t}$ and degrades the beam brilliance. In fact, previous simulation showed that the acceleration field of 10-100 MV/ $\mathrm{m}$ (typically used for electron guns) is sufficient to maintain the low emittance of field emission beamlet with the tip current of $1-10 \mu \mathrm{A} .{ }^{19}$ In contrast, the acceleration field of the present experiment was $\sim 50 \mathrm{kV} / \mathrm{m}$ and not sufficient to maintain the small initial emittance of the space-charge dominated beam, resulting in a larger beam size as observed in Fig. 6. We do not consider that the expanded beam radius at the higher $V_{\text {ge }}$ is due to the nonlinearity of the focusing characteristic of $G_{\mathrm{col}}$ at higher $V_{\mathrm{ge}}$. In fact, observation that the FE1 beam at $V_{\text {ge }}$ of $143 \mathrm{~V}$ (with $\sim 0.1 \mathrm{nA}$ emission current) shown in Fig. 6 is even smaller than the FE2 beam with $V_{\text {ge }}$ of $90 \mathrm{~V}$ indicates that such nonlinearity of the $G_{\mathrm{col}}$ can be small. Nevertheless, $u_{t}$ of the FE2 beam at $V_{\mathrm{ge}}$ of $110 \mathrm{~V}$ and at $k_{\mathrm{col}}$ of 0.94 was small; the value equal to $\sim 0.7 \times 10^{-3} c$ ( $c$ is the light velocity in vacuum) is smaller than the state-of-the-art photocathodes (with intrinsic beam emittance of $0.2 \mathrm{~mm}$ mrad for a $1 \mathrm{~mm}$-diameter cathode) currently in use. ${ }^{20} \mathrm{We}$ also note that the emitter position of FE2 is shifted from the $G_{\text {col }}$ center by $\sim 0.8 \mu$ m caused by misalignment during the electron beam lithography process. Therefore, improved single nanotip emitter performance is expected with a smaller offset of the emitter position to realize a further reduction of the minimum beam size and further enhancement of beam current density.

Finally, we discuss the difference of the field emission current of the two emitters at zero $k_{\mathrm{col}}$ (i.e., $1.24 \mathrm{nA}$ for FE1 at $V_{\mathrm{ge}}$ of $130 \mathrm{~V}$ and $4.04 \mu \mathrm{A}$ for FE2 at $V_{\mathrm{ge}}$ of $110 \mathrm{~V}$ ). Considering the fact that $B_{\mathrm{FN}}$ of the two devices are approximately the same, the lower current and lower $A_{\mathrm{FN}}$ value of FE1 than those of FE2 is most likely ascribed to the difference of the effective emitting area $S$. From the Fowler-Nordheim equation, we can relate the value of $A_{\mathrm{FN}}$ with $S$ using the following approximate relationship (see Ref. 21):

$$
A_{\mathrm{FN}} \approx \mathrm{Sa} b^{2} \Phi^{2} \exp \left(b c^{2} / \Phi^{0.5}\right),
$$

where the constants $a, b$, and $c$ are given by $a$ $=1.541434 \times 10^{-6} \mathrm{AeV} \mathrm{V}^{-2}, \quad b=6.830890 \mathrm{eV}^{-3 / 2} \mathrm{~V} \mathrm{~nm}^{-1}$, and $c=1.199985 \mathrm{eV} \mathrm{V}^{-1 / 2} \mathrm{~nm}^{1 / 2}$, and $\Phi$ is the work function of Mo equal to $4.5 \mathrm{eV}^{21,22}$ Substituting $\pi R_{\text {tip }}{ }^{2}$ for $S, A_{\mathrm{FN}}$ takes the value equal to $0.2-20 \mathrm{~A}$ for $R_{\text {tip }}$ in the range of $1-10 \mathrm{~nm}$. This is in good agreement with $A_{\mathrm{FN}}(=2.95 \mathrm{~A})$ of FE2, obtained from the fitting of the $I-V$, Fig. 2. Accordingly, the small $A_{\mathrm{FN}}$ of FE1 indicates that the emission area of the FE1 beam is much smaller than the emitter tip apex. This interpretation is compatible with the highly nonuniform beam image of FE1 (Fig. 3) with the indication that the active emission part of FE1 is limited to two separate small spots, perhaps occupying a small fraction of $\sim 10^{-2}$ or 
lower at the tip apex. The numerical disagreement of the actual emission area and the value estimated form the Fowler-Nordheim fitting has been discussed previously. ${ }^{22}$ However, further quantitative comparison will require atomically resolved beam image measurement, resolving the work function nonuniformity as well as more involved theory that takes into account the precise expression of the field emission current density; ${ }^{21}$ therefore, it is out of the scope of the present manuscript.

\section{SUMMARY AND CONCLUSIONS}

In summary, we have studied the field emission beam characteristics of double-gate single metal nanotips with $G_{\text {ext }}$ and $G_{\text {col }}$ electrodes. A possibility to produce highly collimated electron beamlet with the proposed double-gate structure, which was shown previously by array emitters, was demonstrated with single tip emitter in this work. The importance of the electrostatic shielding of the tip apex from the collimation potential, previously discussed with the array experiment, was confirmed using well defined single tip operation. We conclude that an excellent collimation capability with minimal current loss can be attained with double-gate single nanotip emitters, optimized in terms of the $G_{\text {ext }}$ aperture shape and the alignment of $G_{\mathrm{col}}$ aperture. In addition, the single tip experiment here showed that one can produce the highly collimated beamlet with the emission current on the order of $1 \mu \mathrm{A}$, which suggests that one can produce highly collimated field emission beam from double-gate FEA with the emission current in the order of tens of milliamperes with the intrinsic emittance below $\sim 0.1 \mathrm{~mm}$ mrad and that nanotips are highly promising for high brilliance applications such as time resolved electron diffraction studies to provide atomic views of structural dynamics.

\section{ACKNOWLEDGMENTS}

The authors acknowledge J. Lehmann and K. Vogelsang for their help on our device fabrication. This work was partially supported by the Swiss National Science
Foundation Nos. 200020_143428 and 2000021_147101. C.L. thanks the Max Planck Society for financial support.

${ }^{1}$ W. P. Dyke and J. K. Trolan, Phys. Rev. 89, 799 (1953).

${ }^{2}$ C. A. Brau, Nucl. Instrum. Methods Phys. Res. A 407, 1 (1998).

${ }^{3}$ P. Hommelhoff, Y. Sortais, A. Aghjani-Talesh, and M. A. Kasevich, Phys. Rev. Lett. 96, 077401 (2006).

${ }^{4}$ C. Ropers, D. R. Solli, C. P. Schulz, C. Lienau, and T. Elsaesser, Phys. Rev. Lett. 98, 043907 (2007).

${ }^{5}$ H. Yanagisawa, C. Hafner, P. Dona, M. Klockner, D. Leuenberger, T. Greber, M. Hengsberger, and J. Osterwalder, Phys. Rev. Lett. 103, 257603 (2009).

${ }^{6}$ S. Tsujino, P. Beaud, E. Kirk, T. Vogel, H. Sehr, J. Gobrecht, and A. Wrulich, Appl. Phys. Lett. 92, 193501 (2008).

${ }^{7}$ P. Helfenstein, A. Mustonen, T. Feurer, and S. Tsujino, Appl. Phys. Express 6, 114301 (2013).

${ }^{8}$ P. Helfenstein, K. Jefimovs, E. Kirk, C. Escher, H.-W. Fink, and S. Tsujino, J. Appl. Phys. 113, 043306 (2013).

${ }^{9}$ S. Tsujino, M. Paraliev, E. Kirk, T. Vogel, F. Le Pimpec, C. Gough, S. Ivkovic, and H.-H. Braun, J. Vac. Sci. Technol. B 29, 02B117 (2011).

${ }^{10}$ S. Tsujino and M. Paraliev, J. Vac. Sci. Technol. B 32, 02B103 (2014).

${ }^{11}$ Y. Neo et al., J. Vac. Sci. Technol. B 27, 701 (2009).

${ }^{12}$ C. M. Tang, M. Goldstein, T. A. Swyden, and J. E. Walsh, Nucl. Instrum. Methods Phys. Res. A 358, 7 (1995).

${ }^{13}$ P. Helfenstein, K. Jefimovs, E. Kirk, C. Escher, H. W. Fink, and S. Tsujino, J. Appl. Phys. 112, 093307 (2012).

${ }^{14}$ V. Guzenko, A. Mustonen, P. Helfenstein, E. Kirk, and S. Tsujino, Microelectron. Eng. 111, 114 (2013).

${ }^{15}$ E. Kirk, S. Tsujino, T. Vogel, K. Jefimovs, J. Gobrecht, and A. Wrulich, J. Vac. Sci. Technol. B 27, 1813 (2009).

${ }^{16}$ A. Mustonen, V. Guzenko, C. Spreu, T. Feurer, and S. Tsujino, Nanotechnology 25, 085203 (2014).

${ }^{17}$ From the analysis of the beam radius of the un-collimated beam (Fig. 6), the estimated beam brilliance of the un-collimated beam with the emission current of $4 \mu \mathrm{A}$ is equal to $\sim 4 \times 10^{10} \mathrm{~A} / \mathrm{cm}^{2}$ str and high, see Handbook of Charged Beam Optics, 2nd ed., edited by J. Orloff (Taylor \& Francis, UK, 2009), p. 440.

${ }^{18}$ P. Das Kanungo, P. Helfenstein, V. A. Guzenko, C. Lee, M. Paraliev, and S. Tsujino, "Electron beam collimation from an all-metal double-gate 40,000 nano-tip array: Improved emission current and beam uniformity upon neon gas conditioning," JVST (submitted).

${ }^{19}$ M. Dehler, A. Candel, and E. Gjonaj, J. Vac. Sci. Technol. B 24, 892 (2006).

${ }^{20}$ Y. Ding et al., Phys. Rev. Lett. 102, 254801 (2009).

${ }^{21}$ R. G. Forbes and J. H. B. Deane, Proc. R. Soc. A 463, 2907 (2007).

${ }^{22}$ C. A. Spindt, I. Brodie, L. Humphrey, and E. R. Westerberg, J. Appl. Phys. 47, 5248 (1976). 